\title{
El cigarrillo electrónico podría ser más efectivo para dejar de fumar que el reemplazo con nicotina
}

Electronic cigarette would be more effective than nicotine replacement to stop smoking

Hajek P y col. N Engl J Med 2019.380; 629-37

\section{Objetivos}

Evaluar la eficacia de los cigarrillos electrónicos recargables en comparación con el reemplazo de nicotina en personas adultas que buscan ayuda para dejar de fumar.

\section{Diseño, lugar y población}

Ensayo clínico aleatorizado y controlado, pragmático, multicéntrico, llevado a cabo en el Reino Unido. Fueron invitadas a participar personas fumadoras adultas que consultaron en tres centros del programa para dejar de fumar del Servicio Nacional de Salud de ese país. Se seleccionaron los pacientes que no manifestaron una fuerte preferencia por la terapia de reemplazo de nicotina los cigarrillos electrónicos, y actualmente no estuviesen usando ningún tipo de producto para dejar de fumar. Se excluyeron a las personas embarazadas o en periodo de lactancia.

\section{Intervención}

Los pacientes fueron asignados al azar a uno de dos grupos: terapia de reemplazo de nicotina de su elección (incluidas las combinaciones de productos) $(\mathrm{N}=446)$; o un paquete de inicio de cigarrillos electrónicos recargable de segunda generación $(\mathrm{N}=438)$. El tratamiento incluyó apoyo conductual semanal durante al menos 4 semanas en ambos grupos. Por la naturaleza de la intervención, los participantes, el equipo de estudio y los evaluadores de resultado conocían la rama de tratamiento asignado, y sólo se pudo mantener el ciego para las personas que analizaron los datos.

\section{Medición de Resultados Principales}

El resultado primario fue la abstinencia sostenida durante un año, medida por autoinforme y definida como el consumo de menos de cinco cigarrillos por día. Este desenlace se validó bioquímicamente mediante la medición de monóxido de carbono (CO) en el aire espirado, con un valor de corte de 8 ppm en la visita final. Los resultados secundarios incluyeron abstinencia secundaria a las cuatro y las 26 semanas, la reducción del consumo de cigarrillos al $50 \%$, el uso del tratamiento informado por los participantes, y los efectos adversos, entre otros. Se realizó el análisis por intención de tratar.

\section{Resultados Principales}

Los resultados principales se resumen en la tabla 1. Se documentó que al año de seguimiento, el producto asignado seguía siendo utilizado por 63 de 79 pacientes (80\%) que lograron la abstinencia en el grupo de cigarrillo electrónico, y por 4 de 44 pacientes $(9 \%)$ en el grupo de reemplazo de nicotina. Los eventos adversos más frecuentes (náuseas e irritación de vías aéreas) fueron leves en ambos grupos.

Tabla 1. Tasas de abstinencia del tabaquismo al año de seguimiento

\begin{tabular}{|c|c|c|c|c|}
\hline Desenlace primario & $\begin{array}{c}\text { Cigarrillo electrónico } \\
\mathbf{n}(\%)\end{array}$ & $\begin{array}{c}\text { Reemplazo de } \\
\text { nicotina } \\
\mathbf{n}(\%)\end{array}$ & $\begin{array}{c}\text { Riesgo relativo } \\
\text { (IC 95\%) }\end{array}$ & $\begin{array}{c}\text { NNT } \\
\text { (IC 95\%) }\end{array}$ \\
\hline Abstinencia & $79(18)$ & $44(9,9)$ & $1,83(1,30$ a 2,58$)$ & $12(8$ a 27$)$ \\
\hline
\end{tabular}

IC: intervalo de confianza; NNT: número necesario a tratar

\section{Conclusiones}

En este ensayo aleatorizado pragmático, los cigarrillos electrónicos fueron más efectivos para dejar de fumar que la terapia de reemplazo de nicotina.
Fuente de financiamiento/conflictos de interés: Instituto Nacional de Investigación en Salud (NIHR), Programa de Evaluación de Tecnología de la Salud (proyecto12/167/135) y subvención A16893 Unidad de Ensayos de Prevención, Investigación en Cáncer del Reino Unido. Sin Conflicto de Interés de los autores.

\section{Comentario}

El cigarrillo electrónico es un dispositivo de vaporización de nicotina que está siendo cada vez más usado en Argentina y en el mundo. Si bien la Administración de Drogas y Alimentos de los EE.UU. (en inglés, FDA) regula la fabricación, la importación, el empaque, la publicidad, la promoción, la venta y la distribución de todos los productos de tabaco, entre ellos, el cigarrillo electrónico, el uso de este dispositivo no tiene una aprobación formal para el tratamiento de cesación tabáquica'.

En Argentina, el cigarrillo electrónico está prohibido por la resolución número 3.226 de la ANMAT desde 2011, al igual que en otros países como Canadá, Brasil, Nueva Zelanda, Uruguay, Colombia y México. A su vez, en 2016 esta agencia regulatoria ratificó su decisión, prohibiendo la importación, distribución, comercialización y publicidad o cualquier otra forma de promoción en el territorio nacional, debido a la escasa evidencia del balance de riesgos y beneficios para la salud ${ }^{2}$. Sin embargo, el cigarrillo electrónico es utilizado por los pacientes, quienes en ocasiones consultan con su médico de cabecera sobre su uso para dejar de fumar.

En conjunto, la evidencia sobre los daños potenciales revisada por el Comité de Revisión de los Efectos a la Salud de los Sistemas Electrónicos de Suministro de Nicotina de la Academia Nacio- nales de Ciencias, Ingeniería y Medicina de los EE.UU. sugiere que los cigarrillos electrónicos no están exentos de actividad fisiológica en los seres humanos, pero las implicaciones para los efectos a largo plazo sobre la morbilidad y la mortalidad aún no están claras, por el poco tiempo de uso de estos dispositivos. El uso de cigarrillos electrónicos en lugar de cigarrillos convencionales por personas con enfermedades respiratorias pre-existentes podría ser menos perjudicial, ya que el aerosol para cigarrillos electrónicos contiene menos cantidades y niveles más bajos de tóxicos que el humo de los cigarrillos de tabaco combustible. Sin embargo, la exposición a la nicotina y a los tóxicos de la vaporización de aromas y humectantes depende de las características del usuario y del dispositivo ${ }^{3}$. Por otro lado, el uso en adultos de los dispositivos electrónicos no sólo puede exponer a los niños al vapor del cigarrillo electrónico, sino también a modelos de comportamiento adictivo. Existe evidencia sustancial de que el uso de cigarrillos electrónicos por parte de los jóvenes aumenta el riesgo de fumar cigarrillos de tabaco ${ }^{4}$, y ya se mencionan en varias publicaciones el aumento exponencial (más del doble entre 2013 y 2014) del uso de estos dispositivos por niños, jóvenes y adultos jóvenes que no han utilizado cigarrillos de tabaco combustible. 
Los datos confiables sobre la eficacia del cigarrillo electrónico como herramienta para dejar de fumar también son limitados. Diversos estudios observacionales, intrínsecamente sujetos a riesgo de sesgo de notificación y factores confusión, han arrojado resultados contradictorios ${ }^{5-7}$. Una revisión Cochrane mostró que los cigarrillos electrónicos con nicotina fueron más efectivos para dejar de fumar que los cigarrillos electrónicos sin nicotina ${ }^{8}$, mientras que otro que comparó los cigarrillos electrónicos con los parches de nicotina mostró cifras bajas de eficacia, similares para ambos herramientas terapéuticas ${ }^{9}$. Resulta importante aclarar que actualmente están ingresando en el mercado otros dispositivos similares al cigarrillo electrónico, denominados "heat not born", pero que utilizan diferente tecnología y por lo tanto estas investigaciones no constituyen evidencia que permita valorar su eficacia y su seguridad.

En cuanto al ensayo clínico que describimos en este resumen, podemos destacar como debilidad metodológica la elección del desenlace principal, ya que se evaluó la abstinencia prevalente cuando lo preferible para este tipo de estudios hubiese sido la abstinencia continua, determinada cuando el paciente permanece sin fumar cigarrillos desde que puso el día $\mathrm{D}$, es decir, no fumó durante todo el tiempo de seguimiento. Adicionalmente, la determinación de $\mathrm{CO}$ en el aire exhalado elegida como método de validación se logra con 24 a 48 hs de abstinencia, lo que podría resultar poco confiable.

Por otro lado, resulta preocupante que una proporción significativa de individuos que lograron la abstinencia en el grupo cigarrillo electrónico continuaran usando el dispositivo al año de seguimiento, motivo por el cual sus detractores aducen que la eficacia que se le atribuye involucra el reemplazo de una adicción por otra.

Otro aspecto a tener en cuenta en este estudio es que no está comparando el cigarrillo electrónico con otros tratamientos aprobados por la FDA, como por ej. el bupropion, que logra tasas de abstinencia de alrededor de $25 \%$ a los seis meses y $20 \%$ a un año de seguimiento ${ }^{10}$, con tasas de abstinencia ligeramente más altas para la terapia combinada que para la monoterapia ${ }^{11}$.

\section{Conclusiones de los comentadores}

Considerando los resultados de este estudio, si el cigarrillo electrónico fuera aprobado por la agencia regulatoria local, podría ser utilizado como estrategia de reducción de daño para pacientes que no pudieron dejar de fumar o que tengan contraindicaciones para utilizar otras terapias farmacológicas.

Ma. Florencia Herrera Bornes [ Servicio de Medicina Familiar y Comunitaria, Hospital Italiano de Buenos Aires ] y Guillermo Espinosa [ Servicio de Medicina Familiar y Comunitaria y GRANTAHI, Hospital Italiano de Buenos Aires, guillermo.espinosa@ hospitalitaliano.org.ar ]

Herrera Bornes, MF y Espinosa G. El cigarrillo electrónico podría ser más efectivo para dejar de fumar que el reemplazo con nicotina. Comentado de: Hajek P y col. A randomized trial of e-cigarettes versus nicotine- replacement therapy. N Engl J Med 2019;380:629-37. PMID 30699054

\section{Referencias:}

1. U.S. Food and Drug Administration. Vaporizadores, cigarrillos electrónicos y otros Sistemas Electrónicos de Administración de Nicotina (ENDS) [Internet]. Citado 6 Mar 2019. Disponible en:

https://esp.fda.gov/tobaccoproducts/labeling/productsingredientscomponents/ucm456610.htm

2. Videla y col. Guía de lectura rápida para el equipo de salud del cigarrillo electronico [Internet]. Ministerio de Salud de la Nación Argentina, Ciudad Autónoma de Buenos Aires ; 2017. Citado 6 Mar 2019. Disponible en: http://www.msal.gob.ar/images/stories/bes/graficos/0000000973cnt-2018-01-22_ guia-rapida-cigarrillo-electronico.pdf

3. Departamento de Salud y Servicios Humanos de los EE. UU. Uso de cigarrillos electrónicos entre los jóvenes y adultos jóvenes [Internet]. Citado 6 Mar 2019. Disponible en: https://e-cigarettes.surgeongeneral.gov/documents/SGR16_Spanish\%20Fact\%20Sheet.PDF

4. Daynard R. Public health consequences of e-cigarettes: a consensus study report of the National Academies of Sciences, Engineering, and Medicine. Journal of Public Health Policy. 2018;39:379-81

5. Adkison SE y col. Electronic Nicotine Delivery Systems. American Journal of Preventive Medicine. 2013;39:207-15

6. Brown $\mathrm{J}$ y col. Real-world effectiveness of e-cigarettes when used to aid smoking cessation: a cross-sectional population study. Addiction 2014;109(9):1531-40

7. Grana RA y col. A longitudinal analysis of electronic cigarette use and smoking cessation. JAMA Intern Med. 2014;174(5):812-3

8. Hartmann-Boyce J y col. Electronic cigarettes for smoking cessation. Cochrane Database Syst Rev. 2016 Sep;9:CD010216

9. Bullen $\mathrm{C}$ y col. Electronic cigarettes for smoking cessation: a randomised controlled trial. Lancet. 2013;382(9905):1629-37

10. Rosen LJ y col. Diminishing benefit of smoking cessation medications during the first year: a meta-analysis of randomized controlled trials. Addiction. 2018;113: 805-16

11. Windle SB y col. Combination Therapies for Smoking Cessation. American Journal of Preventive Medicine. 2016;51:1060-71

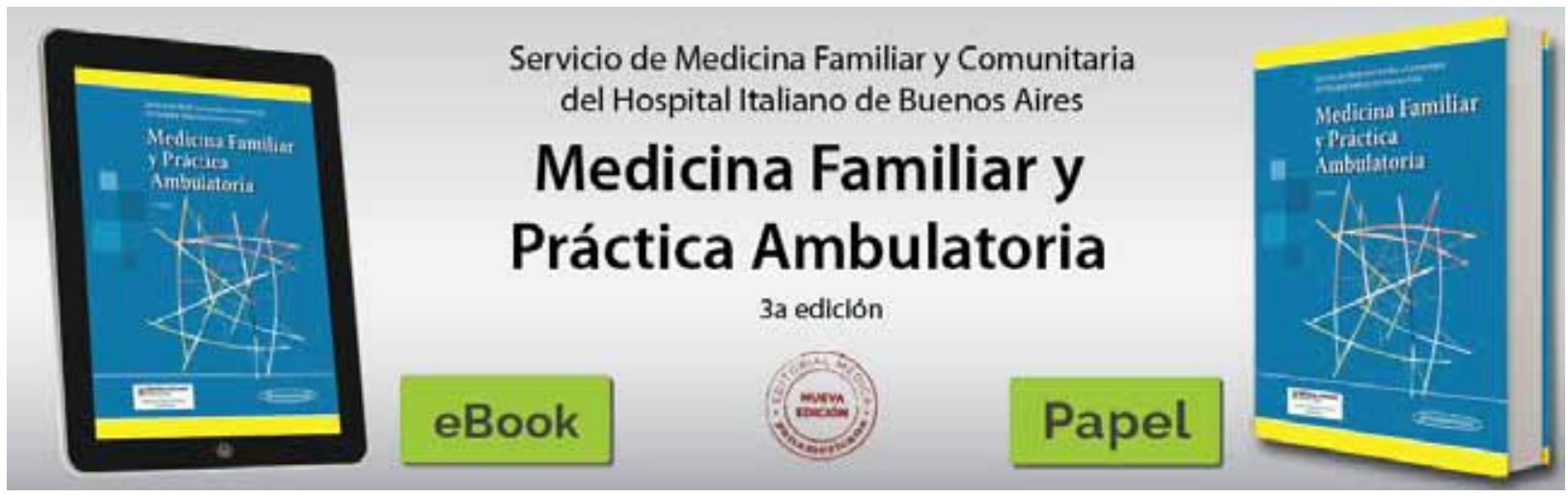

